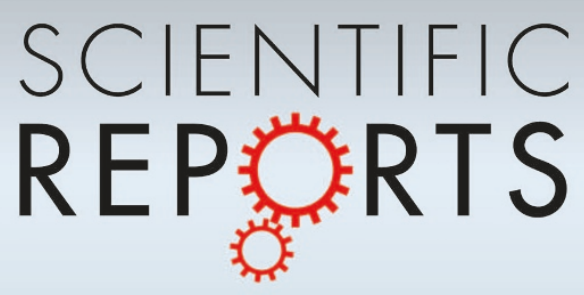

OPEN

SUBJECT AREAS:

MECHANICAL AND STRUCTURAL PROPERTIES

AND DEVICES

SYNTHESIS OF GRAPHENE

ELECTRONIC MATERIALS

ELECTRONIC DEVICES

Received

25 April 2013

Accepted

10 June 2013

Published

25 June 2013

Correspondence and requests for materials should be addressed to L.T.Q. (lqu@bit.edu.cn)

* These authors contributed equally to this work.

\section{Spontaneous Reduction and Assembly of Graphene oxide into Three-Dimensional Graphene Network on Arbitrary Conductive Substrates}

\author{
Chuangang $\mathrm{Hu}^{1 *}$, Xiangquan Zhai ${ }^{*}$, Lili Liu', Yang Zhao', Lan Jiang ${ }^{2} \&$ Liangti Qu'
}

\begin{abstract}
${ }^{1}$ Key Laboratory of Cluster Science, Ministry of Education, School of Chemistry, Beijing Institute of Technology, Beijing 100081, China, ${ }^{2}$ Laser Micro-/Nano-Fabrication Laboratory, School of Mechanical Engineering, Beijing Institute of Technology, Beijing 100081, China.
\end{abstract}

Chemical reduction of graphene oxide (GO) is the main route to produce the mass graphene-based materials with tailored surface chemistry and functions. However, the toxic reducing circumstances, multiple steps, and even incomplete removal of the oxygen-containing groups were involved, and the produced graphenes existed usually as the assembly-absent precipitates. Herein, a substrate-assisted reduction and assembly of GO (SARA-GO) method was developed for spontaneous formation of 3D graphene network on arbitrary conductive substrates including active and inert metals, semiconducting $\mathrm{Si}$, nonmetallic carbon, and even indium-tin oxide glass without any additional reducing agents. The SARA-GO process offers a facile, efficient approach for constructing unique graphene assemblies such as microtubes, multi-channel networks, micropatterns, and allows the fabrication of high-performance binder-free rechargeable lithium-ion batteries. The versatile SARD-GO method significantly improves the processablity of graphenes, which could thus benefit many important applications in sensors and energy-related devices.

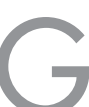
raphene, an atomic-thick layer of carbon atoms with unique properties such as high electrical conductivity, large surface area, good chemical, environmental and mechanical stability, has drawn extensive attention from both the experimental and theoretical communities due to its promising applications in energy, environment, biology, and electronics ${ }^{1-6}$.

For preparation of graphenes, chemical reduction of graphene oxide (GO) is one of the most important routes owing to its feasibility for mass production of graphene-based materials with tailored surface chemistry and functions ${ }^{7}$. However, chemical treatment of GO was usually carried out in reducing circumstances such as hydrazine $\left(\mathrm{N}_{2} \mathrm{H}_{4}\right)^{8}, \mathrm{H}_{2} / \mathrm{Ar}$ with alcohol under high temperatures ${ }^{9}$, strong alkaline sodium/potassium hydroxide $^{10,11}$, sodium borohydride/sulfuric acid ${ }^{12}$, hydriodic acid ${ }^{13}$, sodium-ammonia solution ${ }^{14}$. Besides that the reduction reactions are often involved in the toxicity of the reducing agents, multiple steps, long reaction time, and even incomplete removal of the oxygen-containing group ${ }^{15}$, the produced graphenes are usually collected as random precipitates or agglomeration lacking of well-confined organization such as three-dimensional (3D) graphene assembles ${ }^{16-19}$, which largely limit their applications in energy-related devices.

The recent findings that $\mathrm{GO}$ can be directly reduced by active metals (e.g., $\mathrm{Al}, \mathrm{Fe}, \mathrm{Cu})^{20-22}$ open the new possibility for controlled assembly of graphenes on conductive substrates. Herein, we demonstrate a powerful, facile but efficient substrate-assisted reduction and assembly of GO (SARA-GO) (Figure 1). The spontaneous reduction of GO and its well-organized assembly into 3D network on arbitrary conductive surfaces such as the active metals of $\mathrm{Zn}, \mathrm{Fe}$, and $\mathrm{Cu}$, inert metals of $\mathrm{Ag}, \mathrm{Pt}$, and $\mathrm{Au}$, semiconducting $\mathrm{Si}$ wafer, nonmetallic carbonbased film, and even indium-tin oxide (ITO) glass are achieved readily without any additional reducing agents. As we shall see later, the versatile SARD-GO method offers an efficient approach for constructing a variety of unique graphene assemblies such as microtubes, four-way pipes, spiral tubes, multi-channel networks, and micropatterns, and allowing the fabrication of high-performance binder-free rechargeable lithium-ion batteries (LIBs) by spontaneously deposition of 3D graphene network on Cu foil as anode. The SARD-GO method significantly improves the versatile process of graphenes, which could thus benefit many important applications of graphenes in sensors and energy-related devices. 

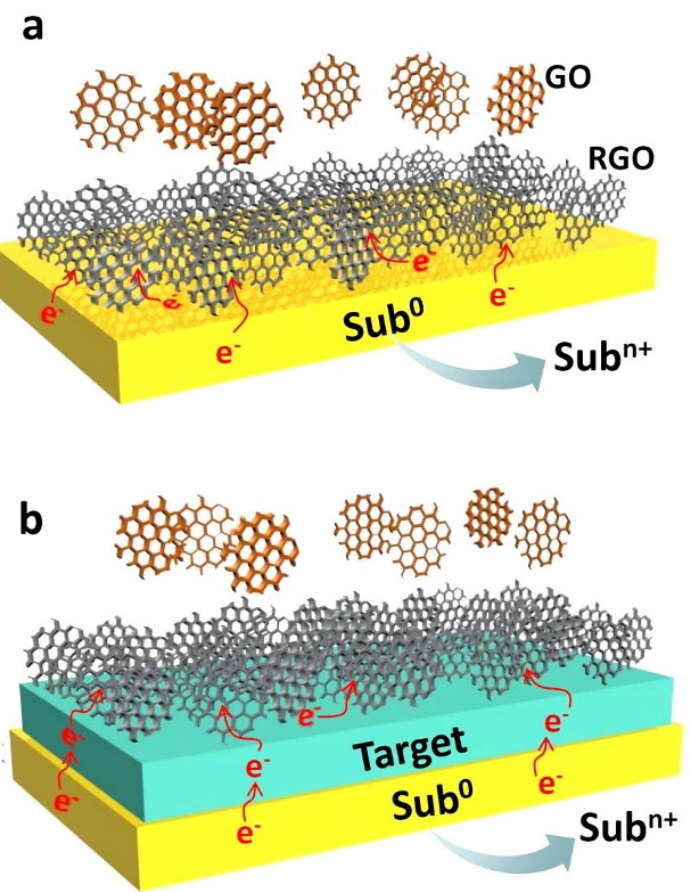

Figure $1 \mid$ SARD-GO process. Schemes of the SARD-GO process on (a) active metal substrate and (b) on the arbitrary conductive target supported by active metal. The active metal substrates $\left(\mathrm{Sub}^{0}\right)$ are oxidized to $\mathrm{Sub}^{\mathrm{n}+}$ ions with electron $\left(\mathrm{e}^{-}\right)$loss, while the GO is reduced by electron reception. The electron transport through the target to the GO allows the 3D assembly of RGO on it.

\section{Results}

SARA-GO on active metal surface. As one example, the $\mathrm{Cu}$ foil has been standing in the aqueous $1 \mathrm{mg} / \mathrm{mL}$ GO suspension with a $\mathrm{pH}=$ 4.0 for several hours without any additives, a black layer of reduced GO (RGO) covers its whole surface (Fig. 2a and Supplementary Fig. S1), which is composed of the $3 \mathrm{D}$ network of RGO sheets (Fig. $2 \mathrm{~b}$ and Supplementary Fig. S2). The amount of deposited RGO on $\mathrm{Cu}$ foil can be modulated conveniently by the reaction time (Supplementary Fig. S3).

TEM investigation reveals that the as-prepared RGO sheets are decorated with nanoparticles (Fig. 2c), which are easily removed after washing with aqueous $\mathrm{HCl}$ solution (Fig. 2d, and Supplementary Fig. S4). Electron diffraction pattern of the RGO sheets presents clear diffraction spots without halation (Fig. 2d, inset), implying the highly crystalline graphene structure.

$\mathrm{X}$-ray diffraction (XRD) further confirms that the representative peak of GO $\left(2 \theta=\mathrm{ca} .11^{\circ}\right.$, the bottom curve in Fig. 2e) has completely disappeared in the as-prepared RGO samples, accompanied with a new broad peak centered at around $24^{\circ}$ and a series of sharp peaks (the middle curve in Fig. 2e). The peaks at $2 \theta=c a .40^{\circ}, 47^{\circ}, 68^{\circ}, 82^{\circ}$, and $86^{\circ}$ correspond to the (111), (200), (220), (311) and (222) planes of face-centered cubic (fcc) structure of $\mathrm{Cu}_{2} \mathrm{O}$ (JCPDS ICDD card NO. 01-1194). After washed with aqueous $\mathrm{HCl}$ solution, the RGO presents a dominant peak centered at $2 \theta=\mathrm{ca} .24^{\circ}$ (the upper curve in Fig. 2e), corresponding to the (002) plane of stacked graphene sheets with an interlayer spacing of about $0.37 \mathrm{~nm}$. It is larger than the $d$ spacing $(0.336 \mathrm{~nm})$ of graphite, suggesting the incompact stacking of as-produced graphenes in the sample.

The chemical structure of the RGO deposited on $\mathrm{Cu}$ foil was also studied by Raman and X-ray photoelectron spectroscopy (XPS). Raman spectrum indicates that GO sheets were reduced and their conjugated structures were partly restored (Supplementary Fig. S5). XPS analysis shows that the intensity of $O$ peak for RGO has decreased drastically in comparison with GO (Fig. 2f). A Cu peak appears on the as-prepared RGO (the middle curve in Fig. 2f) and on the residues in the GO suspension (Supplementary Fig. S6), suggesting the $\mathrm{Cu}$ has been involved in the reduction reaction of GO. The $\mathrm{Cu}$ related peaks vanished in the $\mathrm{RGO}$ sample with aqueous $\mathrm{HCl}$ treatment (Fig. 2f, the upper curve) in consistent with the XRD observation. Fig. $2 \mathrm{~g}$ shows the high resolution $\mathrm{Cu} 2 \mathrm{p}$ spectrum, which reveals that $\mathrm{Cu}$ exists in an oxidized form with two peaks at around 932.2 and $951.9 \mathrm{eV}$ assigned to $\mathrm{Cu} 2 \mathrm{p} 3 / 2$ and $\mathrm{Cu} 2 \mathrm{p} 1 / 2 \mathrm{of} \mathrm{Cu}^{+}$, respectively ${ }^{23,24}$. The XPS also indicates the presence of $\mathrm{Cu}^{2+}$, suggesting that the surfaces of the $\mathrm{Cu}_{2} \mathrm{O}$ nanoparticles were partially oxidized to $\mathrm{CuO}$. The results are consistent with other observations of copper oxidation at the nanoscale ${ }^{25-27}$. Combined with the fact that no diffractions from $\mathrm{CuO}$ were detected in XRD pattern (the middle curve Fig. 2e), it can be concluded that only a small amount of $\mathrm{Cu}_{2} \mathrm{O}$ was oxidized and/or the formed $\mathrm{CuO}$ might be amorphous.

High resolution $\mathrm{C} 1 \mathrm{~s}$ peak of the final RGO proves that oxygencontaining groups have been removed significantly. The $\mathrm{C} 1 \mathrm{~s}$ spectrum of the original GO (Fig. 2h) reveals four types of carbon bonds: $\mathrm{C}=\mathrm{C} / \mathrm{C}-\mathrm{C}(\sim 284.8 \mathrm{eV}), \mathrm{C}-\mathrm{O}$ (hydroxyl and epoxy, $\sim 286.6 \mathrm{eV}$ ), $\mathrm{C}=\mathrm{O}$ (carbonyl, $\sim 287.5 \mathrm{eV}$ ) and $\mathrm{O}-\mathrm{C}=\mathrm{O}$ (carboxyl, $\sim 288.7 \mathrm{eV})^{25}$. For the RGO formed on $\mathrm{Cu}$ foil, however, the majority of oxygen functional groups have been removed and the $\mathrm{C}-\mathrm{C}$ bonds become dominant (Fig. 2h). The $\mathrm{C} / \mathrm{O}$ atomic ratio of RGO is ca. 9.1 (Table S1), which, although not the best, is much higher than that of RGO synthesized by other typical reduction process (Table S2).

Through the extensive investigation mentioned above, we conclude that, based on the SARA-GO in Fig. 1a, GO has been spontaneously reduced to graphene (also called RGO in this work) in a $3 \mathrm{D}$ network form, while the $\mathrm{Cu}$ substrate is oxidized into the $\mathrm{Cu}$ oxide. Other active metals such as $\mathrm{Al}, \mathrm{Zn}, \mathrm{Fe}$, Co also play well for direct formation the 3D graphene network on their foils (Fig. 2i-1 and Supplementary Fig. S7-S16).

SARA-GO on inert metal surface. Although the spontaneous reduction of GO induced by active metals is impressive as demonstrated in current work and others ${ }^{22-24}$, it is not yet applicable to the inert metals such as Ag, Pt and Au (Supplementary Fig. S16), not to mention the nonmetallic conductive substrates. The reduction of $\mathrm{GO}$ and the oxidation of metal substrates are associated with the electron harvest of GO and the electron loss of metal (Fig. 1a), which provides the opportunity to reduce GO onto arbitrary conductive surfaces via SARA-GO process in Fig. 1b. This concept is inspired by the substrate-enhanced electroless deposition (SEED) we developed previously ${ }^{26}$. As schematically shown in Fig. 1b, by simply supporting the inert metals or other conductive surfaces (Target) with an active metal substrate $\left(\mathrm{Sub}^{\circ}\right)$, GO could be reduced and deposited on the Target surface via a process analogous to the galvanic displacement reaction ${ }^{26}$. The inert conductive substrate acts as a "cathode" for GO reduction and deposition of RGO from its suspension, while the active metal foil serves as an "anode" where $\mathrm{Sub}^{0}$ is oxidized into Sub ${ }^{\mathrm{n}+}$. Therefore, the SARA-GO process should allow the assembly of RGO on any conducting substrate as long as a proper base substrate is defined.

To demonstrate this concept, we supported the inert Au sheet (purity: 99.9\%) with the $\mathrm{Cu}$ foil $(\mathrm{Au} / \mathrm{Cu})$ and exposed them to the aqueous GO suspension. As can be seen in Fig. 3b, the Au surface is covered with graphene network just like the porous structure on $\mathrm{Cu}$ foil (Fig. 2b), while the Au sheet without $\mathrm{Cu}$ support remains clear regardless of the reaction time (Fig. 3a). These results confirm the effective deposition of RGO network on inert metal surface via the SARA-GO.

TEM investigation reveals the transparent RGO sheet with clear electron diffraction spots (Fig. $3 c$ and inset) characteristic of the highly crystalline graphene structure. XRD further confirms that the representative peak of $\mathrm{GO}\left(2 \theta \approx 11^{\circ}\right)$ has completely disappeared 
a

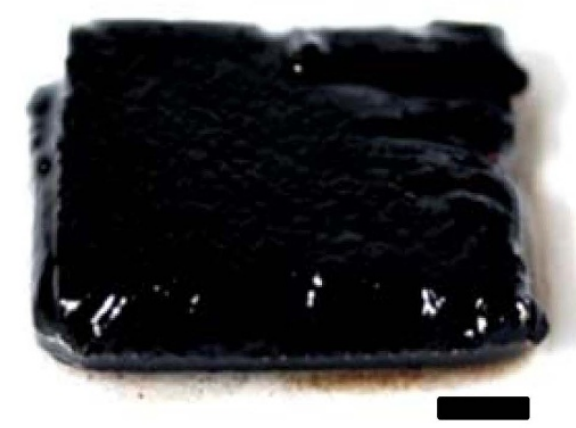

C

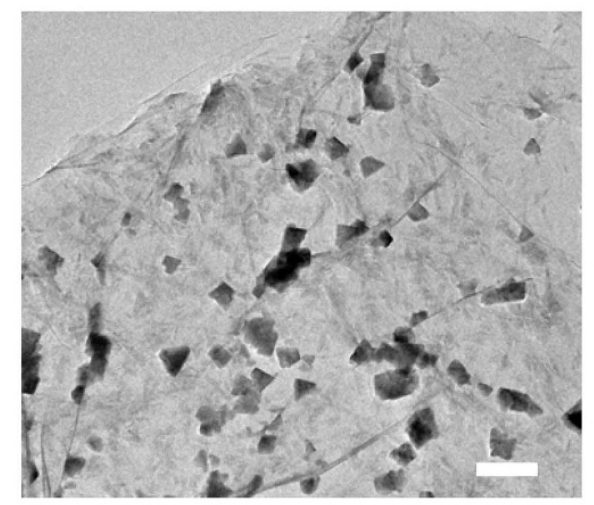

e

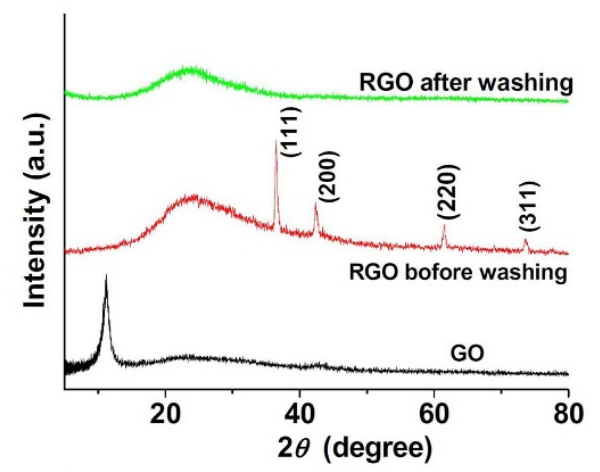

g

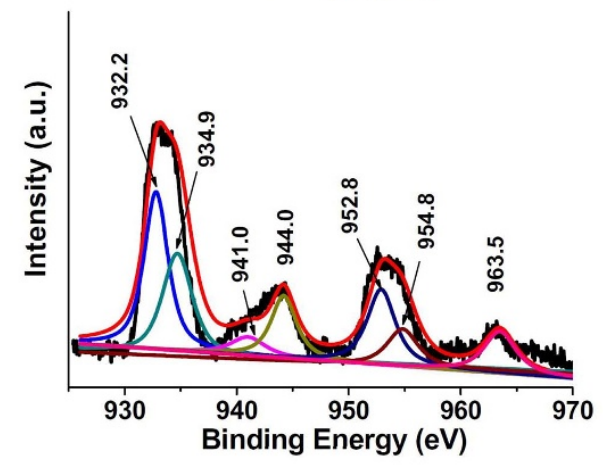

b

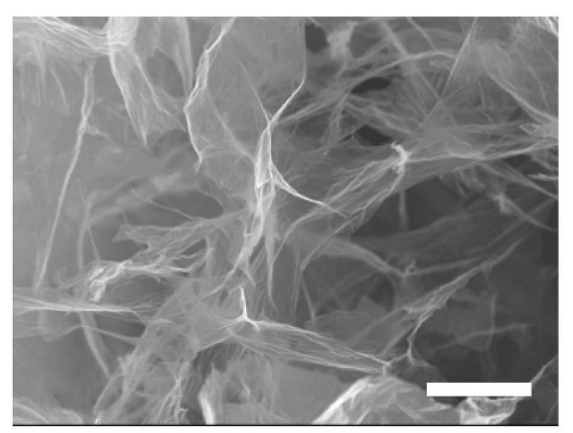

d

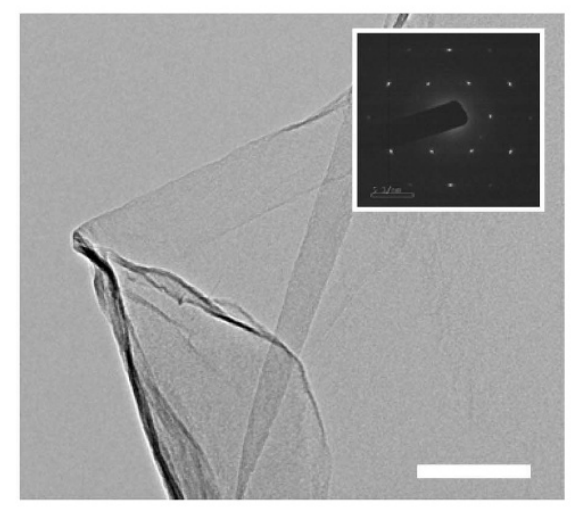

$\mathbf{f}$

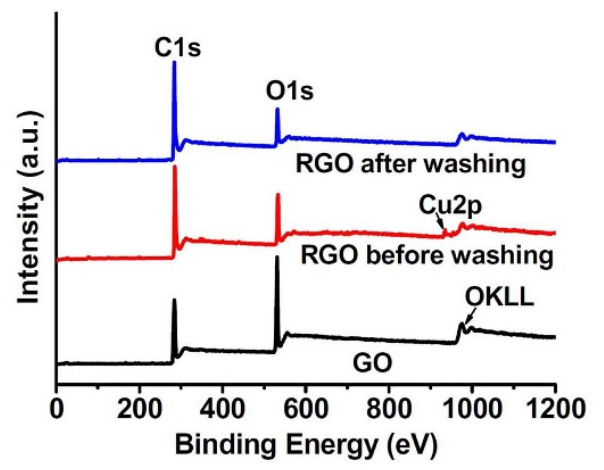

h

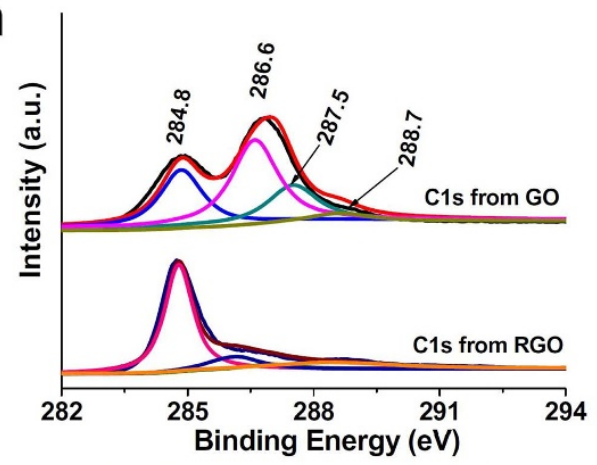

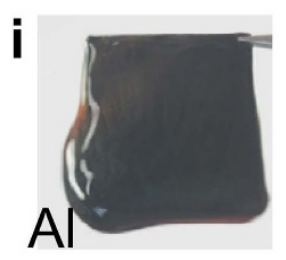
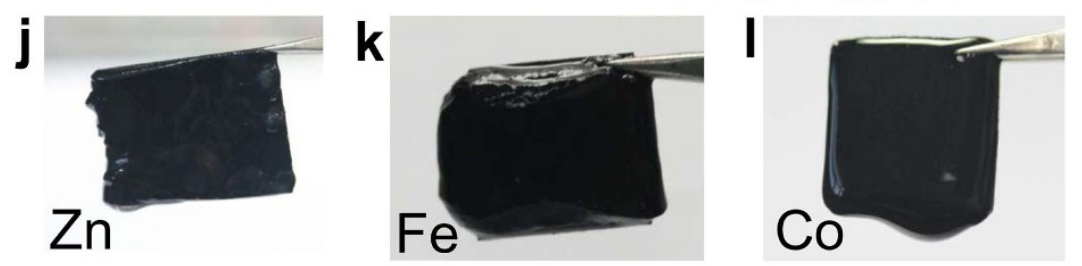

Figure 2 SARA-GO on active metal surface. (a) A photograph of the as-deposited 3D RGO on Cu foil. (b) SEM image of the freeze-dried sample in (a). (c and d) TEM images of RGO on Cu foil before and after treatment with aqueous $\mathrm{HCl}$ solution, respectively. The inset in (d) is the corresponding electron diffraction pattern. (e and f) XRD patterns and XPS spectra of initial GO, as-deposited RGO on Cu before and after washing with aqueous $\mathrm{HCl}$ solution, respectively. (g) High-resolution of $\mathrm{Cu} 2 \mathrm{p}$ peak of RGO as shown in (c) before washing with aqueous $\mathrm{HCl}$ solution, and (h) $\mathrm{Cls}$ peak comparison of GO with RGO after washing with aqueous $\mathrm{HCl}$ solution. (i-l) Photographs of Al, Zn, Fe and Co foils (ca. $\left.2 \times 3 \mathrm{~cm}^{2}\right)$ as-deposited with RGO. Scale bars: (a), $1 \mathrm{~cm}$; (b), $10 \mu \mathrm{m}$; (c), $200 \mathrm{~nm}$; (d), $100 \mathrm{~nm}$. 


\section{a}

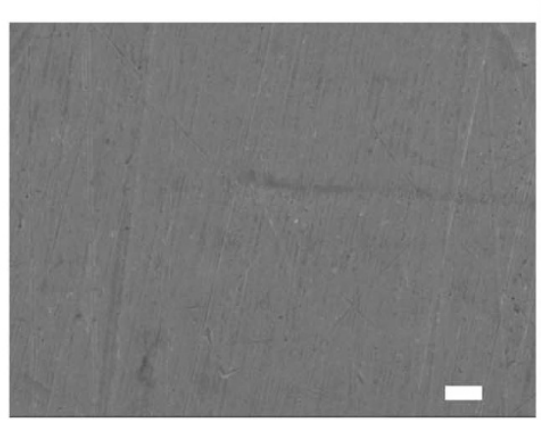

d

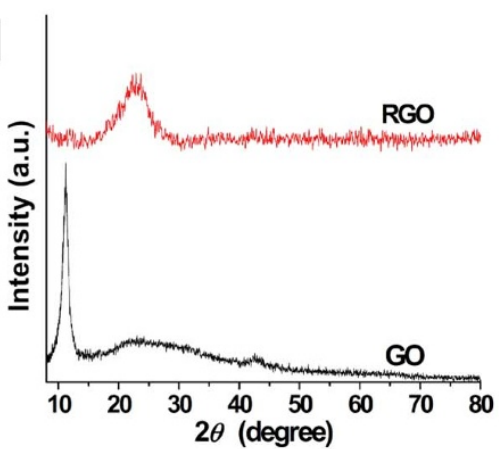

9

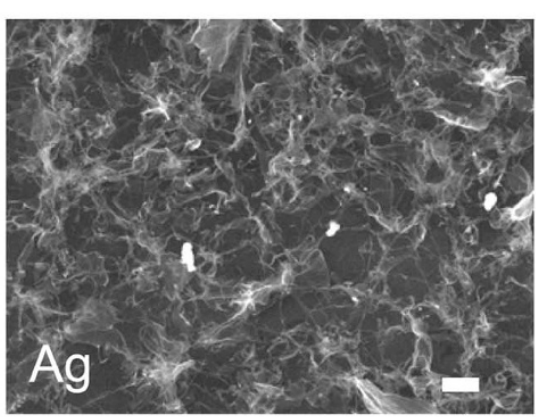

j

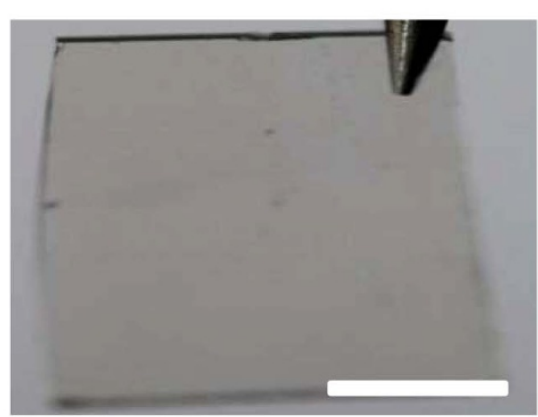

b

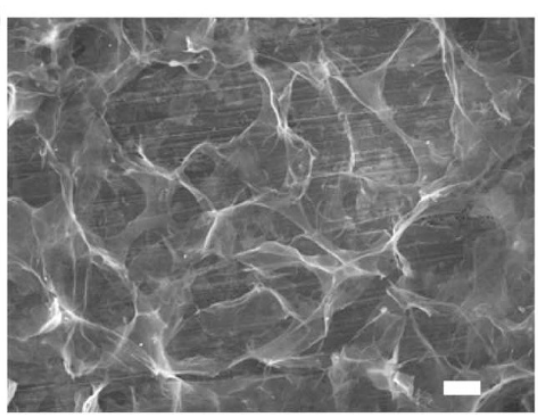

e

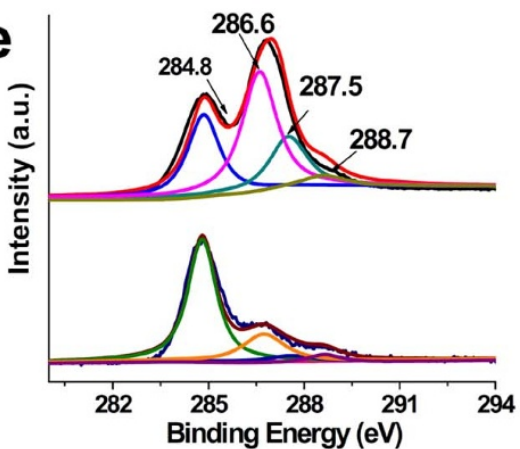

h

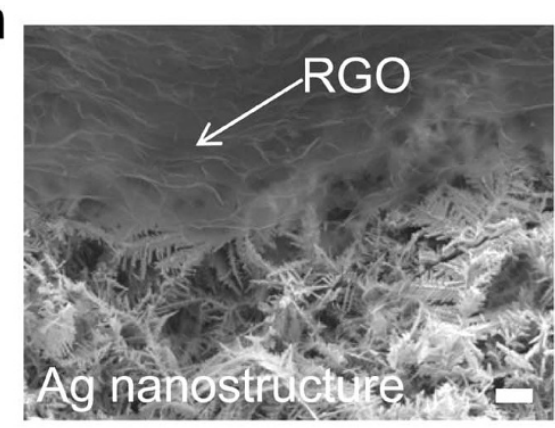

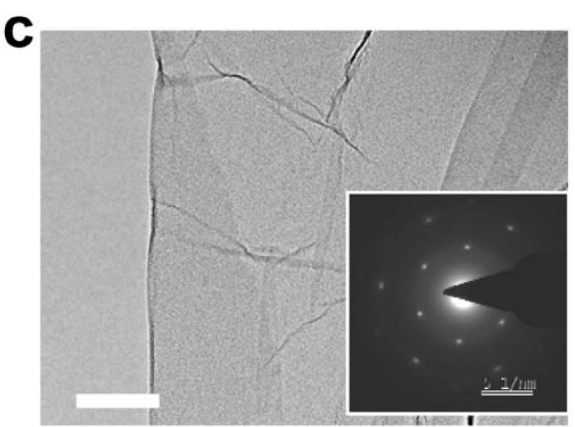
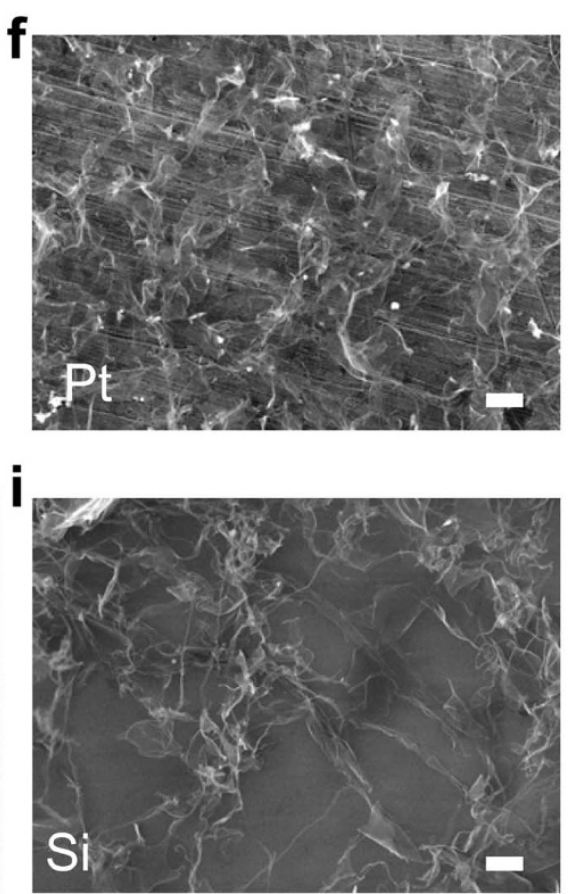

k
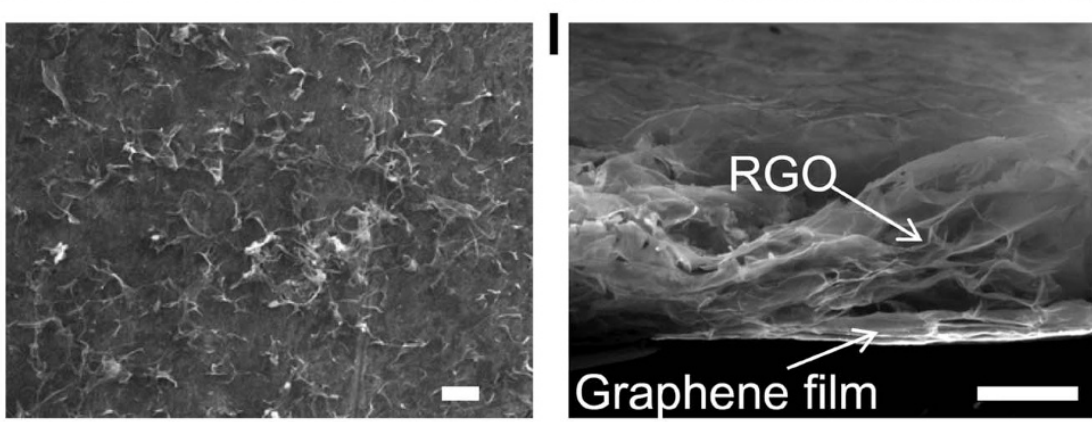

Figure 3 SARA-GO on inert metal surfaces and nonmetallic surfaces. (a and b) SEM images of Au surfaces without and with Cu support after exposure to $1 \mathrm{mg} / \mathrm{mL}$ GO solution and lyophilization, respectively. (c) TEM image and electron diffraction pattern of RGO produced on Au surface supported by $\mathrm{Cu}$ by SARA-GO. ( $\mathrm{d}$ and e) XRD patterns and high-resolution of C $1 \mathrm{~s}$ peaks of RGO produced on Au surface supported by Cu in comparison with initial GO, respectively. ( $\mathrm{f}-\mathrm{h}$ ) SEM images of RGO produced on Pt, Ag and Ag nanostructure surfaces supported with Cu, respectively. (i) SEM image of RGO produced on semiconducting Si supported with $\mathrm{Cu}$. (j and k) Photograph and SEM image of ITO (ca. $2 \times 3 \mathrm{~cm}^{2}$ ) with RGO on it by SARA-GO, respectively. (1) SEM image of RGO produced on graphene film supported by Cu foil. Scale bars: (a, b, f, g, h, i), and (k), $10 \mu \mathrm{m}$; (c), $100 \mathrm{~nm}$; (j), $1 \mathrm{~cm}$; (1), $100 \mu \mathrm{m}$.

in the RGO samples, accompanied with a new broad peak centered at around $23^{\circ}$ for loosely stacked graphenes (Fig. 3d). Accordingly, the C 1s XPS spectra of GO and RGO (Fig. 3e) reflect that most of the oxygenated functional groups of GO have been removed. The $\mathrm{C} / \mathrm{O}$ ratio for the RGO on Au surface is 7.8, in consistent with that of RGO mentioned above (Fig. 2h). These characterizations demonstrate the deposited RGO network on Au surface has a feature of high-quality graphene similar to that on $\mathrm{Cu}$, indicating the feasibility of SARA-GO.
Apart from the $\mathrm{Au}$, the SARA-GO process also works well on such inert metals as $\mathrm{Pt}$ and $\mathrm{Ag}$ when their sheets were supported by $\mathrm{Cu}$ layers (Fig. 3f\&g, and Supplementary Fig. S17). Similarly, RGO network can also be deposited on the Cu covered inert metals, such as $\mathrm{Pt}$, $\mathrm{Ag}$ and $\mathrm{Au}$ (Supplementary Fig. S18).

To further demonstrate the effectiveness of SARA-GO process on the nanostructures of inert metals, we have put the $\mathrm{Au}, \mathrm{Ag}$, and $\mathrm{Pt}$ nanostructures with different sizes and shapes on $\mathrm{Cu}$ foil into the aqueous GO suspension (Supplementary Fig. S19). As expected, they 
behavior as the good substrates to reduce the GO and assemble 3D RGO network on them (Supplementary Fig. S20-S22), indicating the morphology-independent feature of the practicable SARA-GO. Fig. 3h shows the deposited RGO network on Ag nanoleaves.

SARA-GO on nonmetallic surface. More importantly, the SARAGO process allows the RGO spontaneously deposited on any conductive surfaces such as semiconducting Si (Fig. $3 \mathrm{i}$ and Supplementary Fig. S23\&24), ITO glass (Fig. 3j\&k and Supplementary Fig. S25), carbon (Fig. S26) and graphene films (Fig. 31 and Supplementary Fig. S27\&28), indicating the versatile SARA-GO method is universal for formation of graphene network on conductive surfaces for various applications. Specially, the RGO network formed on ITO surface can have no obvious influence on the transparency with a proper reaction time (Fig. 3j\&k and Supplementary Fig. S25).

Fabrication of graphene microtubes, four-way pipe, multichannel network and graphene micropatterns via SARA-GO process. The SARA-GO process allowing the spontaneous reduction of GO on various conductive surfaces offers a facile but efficient method for construction of unique graphene assemblies of practical importance. As exemplified in Fig. $4 \mathrm{a} \& \mathrm{~b}$, the reduction of $\mathrm{GO}$ along a $\mathrm{Cu}$ wire will lead to the formation of graphene microtube $(\mu \mathrm{GT})^{27,28}$. The $\mathrm{Cu}$ wire will act as the support for aggregation of RGO network, and meters of $\mu \mathrm{GT}$ s with a diameter depending on the used $\mathrm{Cu}$ wire are collected after $\mathrm{Cu}$ etching in aqueous $\mathrm{FeCl}_{3} / \mathrm{HCl}$ solution (Fig. 4b, Supplementary Fig. S29-S31). Further, specifically defined configurations such as spiral tubes (Fig. S32), four-way pipe (Fig. 4c, Supplementary Fig. S33), and multi-channel network of graphenes (Fig. 4f, Supplementary Fig. S34) with tunable length and diameter in large scale can be done readily.

Fig. $4 \mathrm{c}$ displays the as-prepared four-way pipe, and the inserts in Fig. $4 \mathrm{c}$ are the SEM images for the four nozzles, indicating the characteristics of hollow interior as the schematic diagram in Fig. 4d. Similarly, $\mathrm{Cu}$ mesh (Fig. 4e), as well as the Fe mesh (Supplementary Fig. S34), will induce the woven fabric of $\mu \mathrm{GTs}$ (Fig. $4 \mathrm{f}-\mathrm{h}$, Supplementary Fig. S34).

The region-defined patterns of RGO network are also available through the powerful SARA-GO. As presented in Fig. 4i and Supplementary Fig. S35, by pressing a Cu grid $(200 \mu \mathrm{m}$ in side length) on Pt foil and exposing them to the GO suspension, we can achieve the RGO patterns on $\mathrm{Pt}$ foil after removing the $\mathrm{Cu}$ grid. The region-confined $3 \mathrm{D}$ graphene network remains well with freezedrying (Fig. 4j). Depending on the Cu masks, a variety of specific patterns on nonconductive substrates can also be designed for various applications (Supplementary Fig. S36\&37).

SARA-GO for construction of binder-free lithium-ion batteries (LIBs). Apart from the construction of unique graphene configurations of practical importance mentioned above, the SARA-GO method enabling the self-organization of 3D graphene network on various conductive substrates will largely facilitates the fabrication of graphene electrodes, and thus benefits the device applications.

As one of the important energy storage devices, rechargeable LIBs have drawn worldwide interest ${ }^{2,3}$. However, graphite, the most commonly used commercial anode material, encounters serious disadvantages such as low theoretical specific capacity $\left(372 \mathrm{mAh} \mathrm{g}^{-1}\right)$ and limited rate capability. As a result, great efforts are being devoted to developing new electrode materials for high performance LIBs. Graphene is expected to be a good candidate as a high-power and high-energy electrode material due to its intrinsically superior electrical conductivity, excellent mechanical flexibility, remarkable thermal conductivity, high surface area ${ }^{29,30}$, and high chemical diffusivity of $\mathrm{Li}, \sim 10^{-7}-10^{-6}\left(\mathrm{~cm}^{2} \mathrm{~s}^{-1}\right)^{31,32}$. Herein, the SARA-GO method provides a straightforward route to assemble graphene network on the $\mathrm{Cu}$ foil in the form of $3 \mathrm{D}$ pore-interconnected structure, which can be directly used as anode electrode in LIBs without need of any ancillary materials such as binders and conductive additives required for the conventional LIB fabrication.

The cropped $\mathrm{Cu}$ foil (Fig. 5a) was first deposited with RGO network on one side via SARA-GO (Fig. 5b). After slightly washing with aqueous $\mathrm{HCl}$ to remove the $\mathrm{Cu}$ oxides, the freeze-dried sample (Fig. 5c) as anode was directly assembled in a coin battery (Fig. 5d).

As shown in Fig. 5e, the RGO/Cu electrode shows typical cyclic voltammogram (CV) curves ( 1 to 5 cycles) of the carbonaceous anode materials at a scan rate of $0.5\left(\mathrm{mV} \mathrm{s}^{-1}\right)$. The peak at around $0.8 \mathrm{~V}$ can be ascribed to the decomposition of the electrolyte on the surface of RGO, leading to the formation of the solid electrolyte interphase (SEI) layer ${ }^{33,34}$. The CV curves of the second to the fifth cycle almost overlap, implying an excellent cycling ability of the $\mathrm{RGO} / \mathrm{Cu}$ electrode.

Fig. $5 \mathrm{f}$ shows the galvanostatic charge/discharge profiles of the RGO/Cu electrode between $0.01-3 \mathrm{~V}$ at a current density of $0.1 \mathrm{~A}$ $\mathrm{g}^{-1}$. The initial discharge and charge specific capacities are 2472 and $1605 \mathrm{mAh} \mathrm{g}^{-1}$, respectively. The irreversible capacity loss is generally attributed to the formation of $\mathrm{SEI}^{33,34}$. The $\mathrm{RGO} / \mathrm{Cu}$ electrode becomes stable at the ninth cycle with a capacity of $1076 \mathrm{mAh} \mathrm{g}^{-1}$, which is about 2.9 times higher than the theoretical one of graphite $\left(372 \mathrm{mAh} \mathrm{g}^{-1}\right)$.

Fig. $5 \mathrm{~g}$ shows the cycling performance and the corresponding coulombic efficiency at a current of $0.3 \mathrm{~A} \mathrm{~g}^{-1}$ after aging at $0.1 \mathrm{~A}$ $\mathrm{g}^{-1}$ for 10 cycles. The discharge capacity stabilizes at $725 \mathrm{mAh} \mathrm{g}^{-1}$ for 60 cycles, which is about 2 times higher than the theoretical capacity of graphite, superior to that of many other carbon materials (Table S3) including carbon nanosprings ${ }^{35}$, carbon nanocages ${ }^{36}$, carbon nanofibers $(\mathrm{CNF})^{37}$, carbon nanotubes $(\mathrm{CNT})^{38}, \mathrm{CNF} \mathrm{CNT}^{39}, \mathrm{~N}$ doped graphene ${ }^{40}$ and N,S-codoped porous graphene ${ }^{41}$, as well as metal oxides of $\mathrm{CoMn}_{2} \mathrm{O}_{4}$ microspheres ${ }^{42}$, and their composites such as $\mathrm{MnO}_{2} / \mathrm{CNT}^{43}$ and $\mathrm{Fe}_{3} \mathrm{O}_{4} / \mathrm{RGO}^{44}$. At the meanwhile, the coulombic efficiency of the battery retained at nearly $100 \%$ during the cycling, showing an excellent reversibility. Furthermore, the 3D RGO/Cubased battery has also a great rate behavior as tested under variable rates from $0.1 \mathrm{~A} \mathrm{~g}^{-1}$ to $20 \mathrm{~A} \mathrm{~g}^{-1}$ (Fig. 5h). Even at $20 \mathrm{~A} \mathrm{~g}^{-1}$, the reversible capacity is still $231 \mathrm{mAh} \mathrm{g}^{-1}$, which is closed to the highly $\mathrm{N}$-doped carbon materials with excellent performance ${ }^{34,45}$. Remarkably, after the high-rate measurements, the specific capacities of the $\mathrm{RGO} / \mathrm{Cu}$ electrode almost recover to the initial values cycled at $100 \mathrm{~mA} \mathrm{~g}^{-1}$. A long-term cycling stability has also been carried out at a high current density of $2 \mathrm{~A} \mathrm{~g}^{-1}$. As shown in Fig. 5i, the anode material shows stable cycling behavior, and the reversible capacity of up to about $500 \mathrm{mAh} \mathrm{g}^{-1}$ is achieved for 300 cycles, which is still ca. $134 \%$ of the theoretical capacity of graphite. On the other hand, the electrochemical impedance spectra of the RGO/Cu electrode before and after cycling at $0.3 \mathrm{~A} \mathrm{~g}^{-1}$ for 100 cycles indicate the activated and improved reaction kinetics upon cycling, and the good contact between the $\mathrm{RGO} / \mathrm{Cu}$ electrode and the current collector (Supplementary Fig. S38).

\section{Discussion}

As we know, GO can be reduced by strong reduction reagents such as hydrazine ${ }^{8}$. However, GO as a strong oxidant is paid the little attention. In fact, GO has the capability to oxidize $\mathrm{Fe}^{2+}$ to $\mathrm{Fe}^{3+}$ in despite of the relatively high redox potential of $+0.77 \mathrm{~V}$ (vs. standard hydrogen electrode, SHE) for $\left(\mathrm{Fe}^{2+} / \mathrm{Fe}^{3+}\right)^{46}$. Example also includes the oxidation of iodic ions $\left(\mathrm{I}^{-}\right)$into neutral iodine $\left(\mathrm{I}_{2}\right)\left(+0.54 \mathrm{~V}^{\mathrm{v}}\right.$. SHE for $\mathrm{I}^{-} /$ $\mathrm{I}_{2}$ ) by GO suspension ${ }^{47}$. Therefore, it is reasonable to observe that GO was reduced by active metals such as $\mathrm{Al}, \mathrm{Fe}, \mathrm{Cu}^{20-22}$.

By curve fitting analysis of the XPS results (Fig. 2h), we can find that the hydroxyl, epoxy and carbonyl groups decrease drastically, while the carboxylic acid groups remain relatively stable. Considering that the epoxy and hydroxyl groups are the main active oxide groups of GO for the typical reduction process ${ }^{48,49}$, and epoxy group can also convert to hydroxyl group in aqueous acidic solution, we 
a

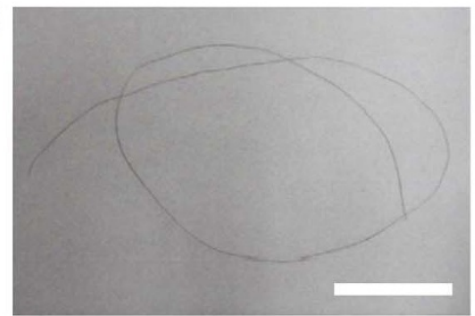

C

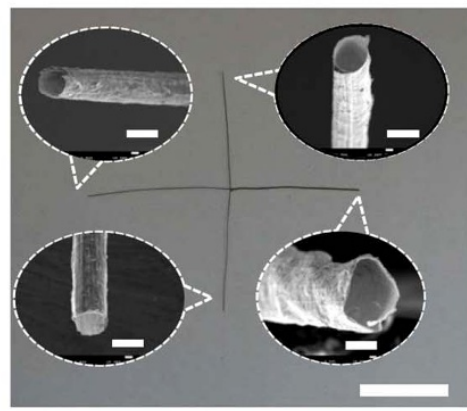

e

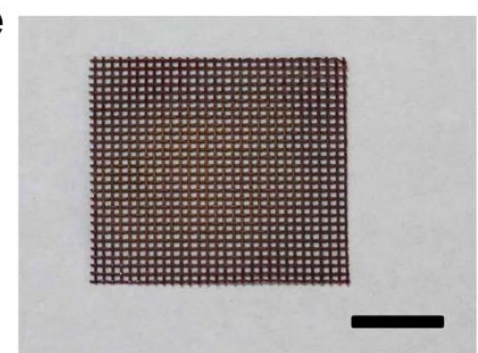

g
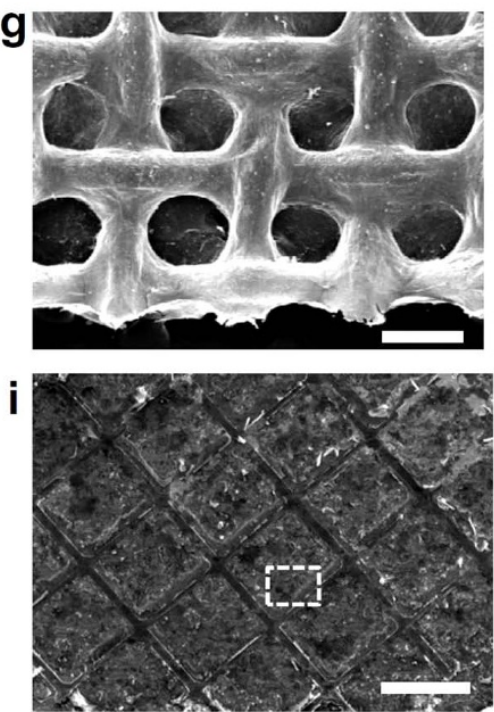

b

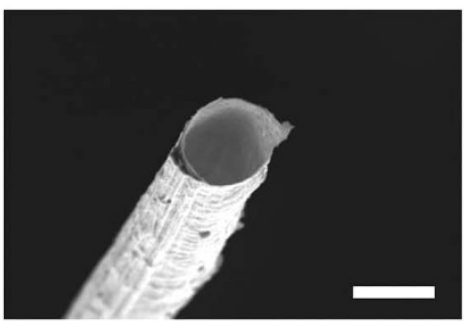

d

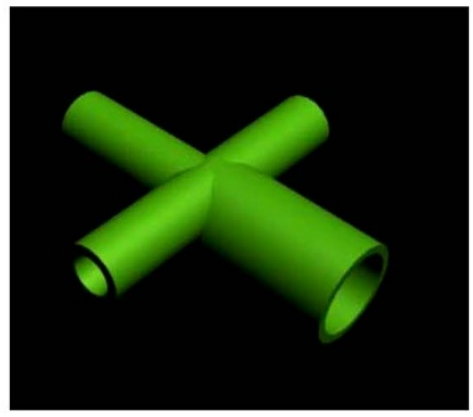

f

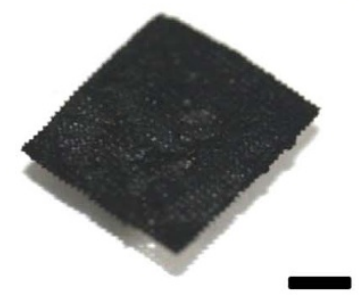

h
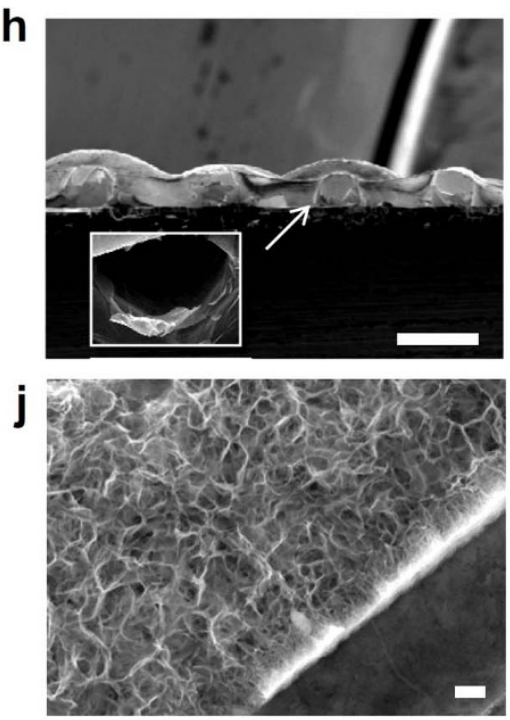

Figure 4 | Fabrication of graphene microtubes, four-way pipe, multi-channel network and graphene micropatterns via SARA-GO process. (a and b) Photograph and SEM image of the graphene microtube produced by SARA-GO on Cu wire. (c) Photograph of a four-way tube with the corresponding SEM images of the nozzles. (d) The corresponding schematic diagram of (c). (e and f) The Cu mesh and the derived graphene mesh by SARA-GO, respectively. ( $g$ and $h$ ) Top and side SEM images of graphene mesh in ( $f$ ), respectively. (i) Graphene patterns on the Pt foil and (j) the enlarged view of the marked area in (i). Scale bars: (a, c, e, f). $1 \mathrm{~cm}$; (b), $50 \mu \mathrm{m}$; (g), (h), $500 \mu \mathrm{m}$; (i), and (c) inset, $100 \mu \mathrm{m}$; (j), $1 \mu \mathrm{m}$.

proposed the reaction mechanism via an electron transfer process of GO with hydroxyl groups as exemplified model in Figure 6. The hydrions are easy to form the complex with the oxygen-related groups in GO, which could induce a dehydration process and allow the positively charged GO system to receive the electrons from the active metals ${ }^{20-22}$. Accordingly, metal ions produced by electron-lost metals could combine the hydroxide $\left(\mathrm{OH}^{-}\right)$ions to balance hydrogen $\left(\mathrm{H}^{+}\right)$. These metastable metal hydroxides then further performed a dehydration process to form metal oxides as we have experimentally observed the formation of such metal oxides as $\mathrm{Cu}_{2} \mathrm{O}$ (Fig. 2c), $\mathrm{ZnO}$ (Supplementary Fig. S7-S9), and $\mathrm{Fe}_{3} \mathrm{O}_{4}$ (Supplementary Fig. S10\&S11) during SARA-GO.

Besides, we also performed the first principle calculation to compare the electron properties of metal surfaces on the basis of the work functions. The work function is the minimum energy required to release electrons from the reactive surface, which should give some 

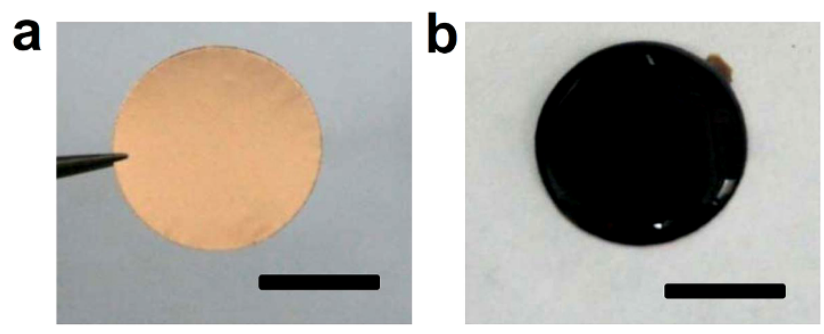

C
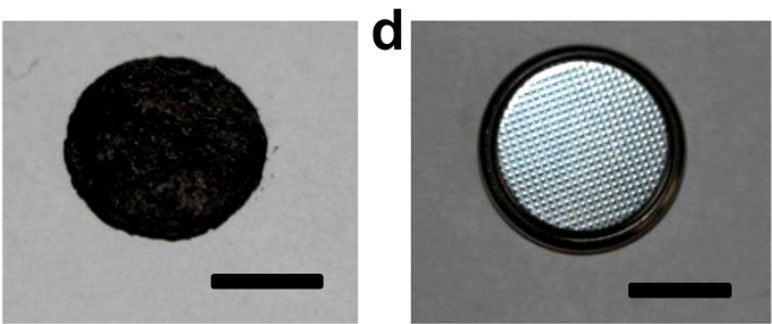

f

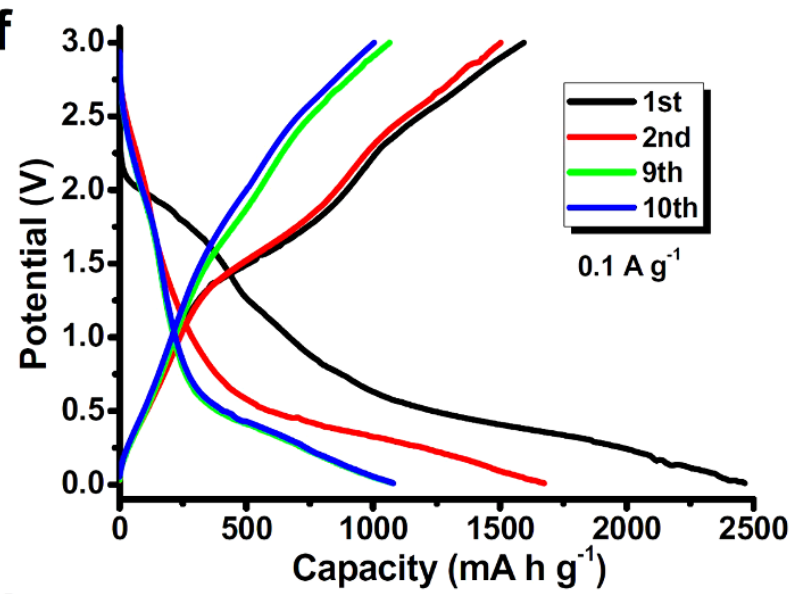

h

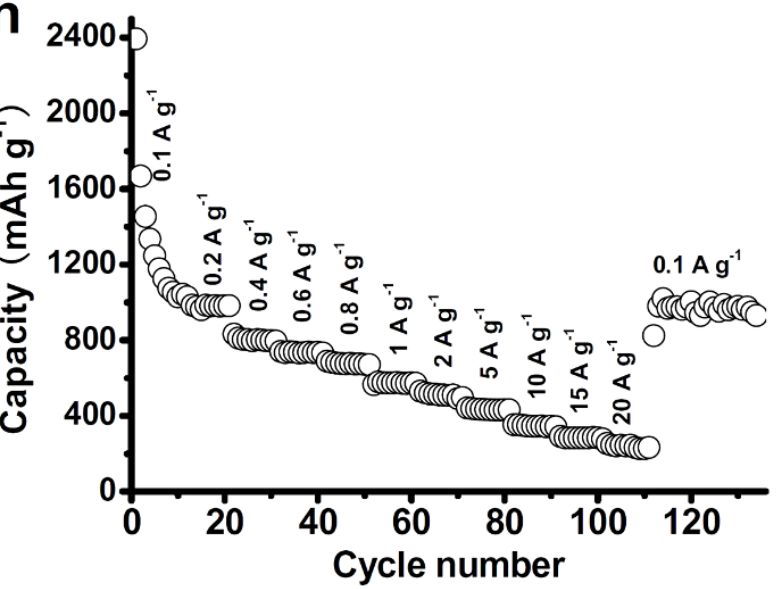

e
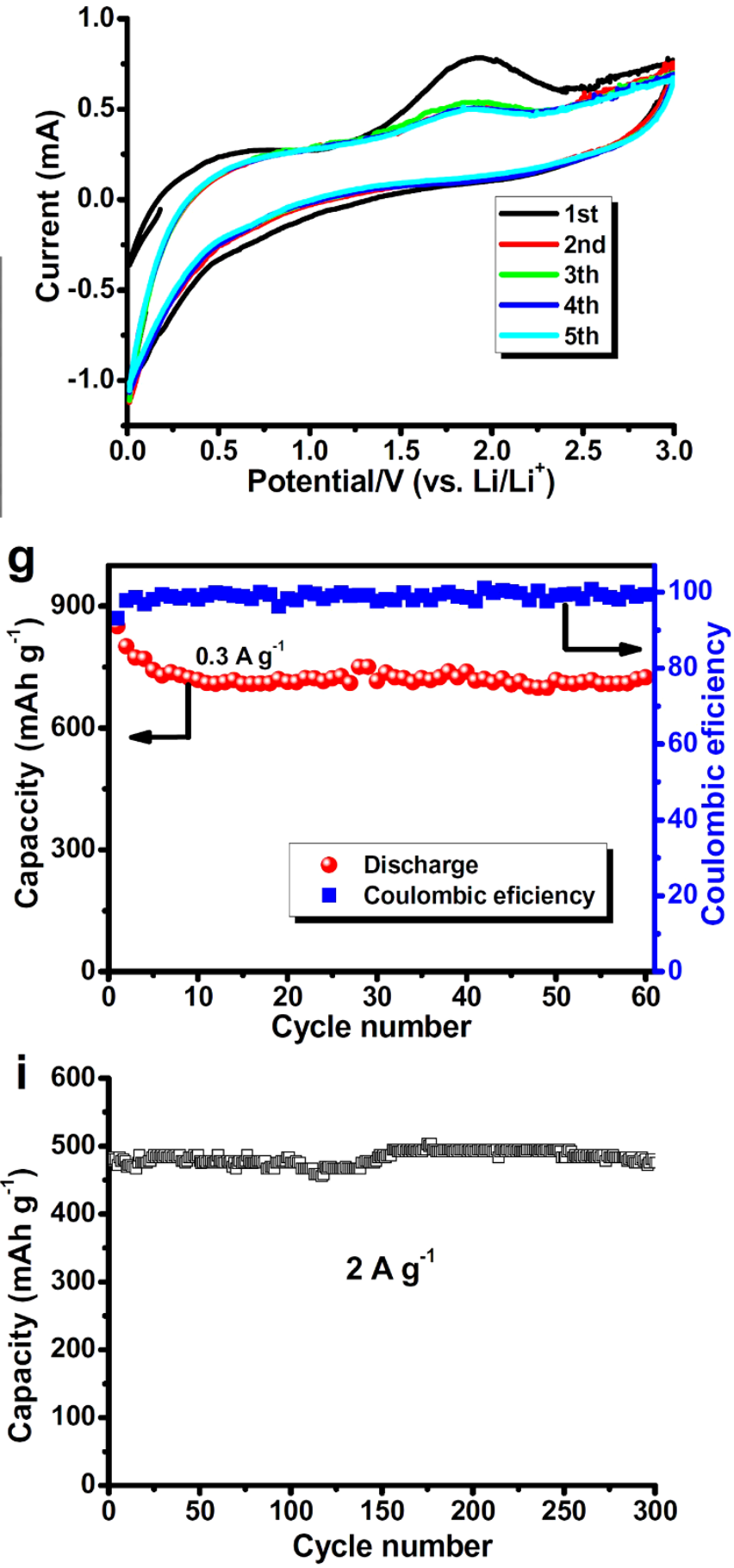

Figure $5 \mid$ Characterization of the binder-free LIBs. (a) Photograph of the trimmed Cu foil with circular shape. (b) RGO assembled on Cu foil by SARA-GO. (c) The 3D RGO formed on Cu foil after lyophilization process. (d) The Li coin battery using the Cu foil supported 3D RGO as anode. (e) CVs of the first five cycles of the RGO/Cu electrode. (f) Charge and discharge voltage profiles for the first, second, ninth, and tenth cycles between 0.01-3.00 V at a current density of $0.1 \mathrm{~A} \mathrm{~g}^{-1}$. (g) Discharge capacity vs. cycle number of the coin battery at a current density of $0.3 \mathrm{~A} \mathrm{~g} \mathrm{~g}^{-1}$ and the corresponding coulombic efficiency. (h) Rate capability test at various current densities. (i) Cycling performance of the coin battery at a current density of $2 \mathrm{Ag}^{-1}$ for 300 cycles. The scale bars in $(\mathrm{a}-\mathrm{d})$ are $1 \mathrm{~cm}$.

clues why different metals have the quite different performances for reducing GO. In our calculation the generalized gradient approximation with the Perdew-Burke-Ernzerhof functional ${ }^{50}$ was used, the basis set contains plane waves up to an energy cutoff of $380 \mathrm{eV}$ and the structure is relaxed until the force on each atom is less than $0.03 \mathrm{eV} / \AA$ in the Vienna ab initio software package (VASP) ${ }^{51,52}$.
We performed the calculation on several metal slabs of $\mathrm{Zn}$ (0001), $\mathrm{Al}$ (111), $\mathrm{Cu}(111), \mathrm{Pt}(111)$ and $\mathrm{Au}$ (111) surfaces with seven layers as examples using Periodic Boundary Conditions (PBC) (Supplementary Fig. S39). The work functions of these metals are $4.02 \mathrm{eV}$, $4.13 \mathrm{eV}, 4.73 \mathrm{eV}, 5.85 \mathrm{eV}$ and $5.01 \mathrm{eV}$, respectively. These results indicate the capacity to lose electrons of the different metals. 


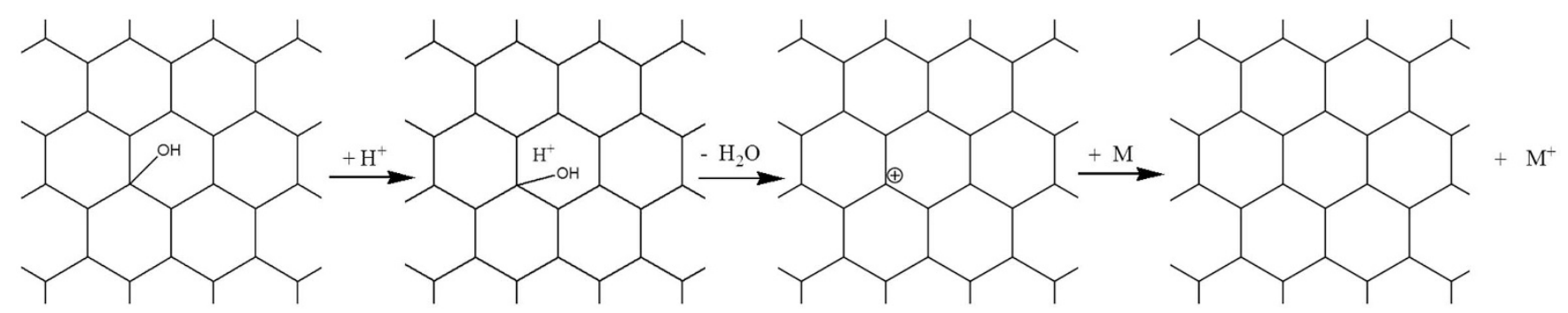

Figure 6 The proposed reaction mechanism. The hydrion forms a complex with the oxide groups (e.g., hydroxyl group) in GO. After the dehydration process, the positively charged GO gets the electrons from the active metal (M) and the metal is oxidized to $\mathrm{M}^{+}$ions. Only one electron transfer is presented in this schematic diagram.

Obviously, the active metals like $\mathrm{Zn}, \mathrm{Al}$ and $\mathrm{Cu}$ are much more active than Pt and Au for GO reduction in consistent with the experimental observation. Although the difficulty to directly reduce GO on inert metals of Pt and Au, the SARA-GO (Figure 1b) developed in this study provide the solution.

It is also notable that the SARA-GO method will generate the graphene assemblies with easy in-situ functionalization. As can be seen in Fig. 2c and Supplementary Fig. S7-S11, the functional components such as $\mathrm{Cu}_{2} \mathrm{O}, \mathrm{ZnO}$ and $\mathrm{Fe}_{3} \mathrm{O}_{4}$, if not washed away by aqueous $\mathrm{HCl}$, could be spontaneously attached on the graphene sheets during the SARA-GO process. Therefore, new functions could be expected due to these incorporated components. As an example shown in Supplementary Fig. S40, the as-prepared $\mathrm{ZnO}$-containing graphene film presents the fast photocurrent response with good repeatability upon exposure to a daylight lamp (100 W).

In conclusion, a general method of substrate-assisted reduction and assembly of graphene oxide has been developed for spontaneous deposition of 3D graphene network on arbitrary conductive substrates without any additional reducing agent. The SARA-GO process offers a facile, but efficient approach for constructing a variety of unique graphene assemblies of practical importance. For examples, graphene microtubes, four-way pipes, spiral tubes, multi-channel networks, and micropatterns have been conveniently manufactured by using $\mathrm{Cu}$ or Fe wires and meshes as templates. The SARA-GO enabling the self-organization of $3 \mathrm{D}$ graphene network on various conductive substrates largely facilitates the fabrication of graphene electrodes. The binder-free LIBs using spontaneously deposited 3D graphene network on $\mathrm{Cu}$ foil as anode materials have been demonstrated, which exhibit high capacity, cycling stability and rate performance superior to many other carbon-based materials and metal oxide composites. The versatile SARD-GO method with significantly improved processability of graphenes will benefit a variety of applications beyond those specifically demonstrated in this preliminary study.

\section{Methods}

Synthesis of Graphene Oxide (GO). GO was prepared from natural graphite powder via acid-oxidation according to a modified Hummers method as mentioned in our previous papers $^{53,54}$.

Spontaneous deposition of 3D RGO network on $\mathrm{Al}, \mathrm{Zn}, \mathrm{Fe}, \mathrm{Co}, \mathrm{Cu}$ foils. $\mathrm{Zn}, \mathrm{Fe}$, $\mathrm{Cu}, \mathrm{Co}$ and $\mathrm{Al}$ foils were exposure to $1 \mathrm{mg} / \mathrm{mL}$ GO solution for a certain time (e.g, 1$12 \mathrm{~h}$ ) at ambient condition (Supplementary Fig. S1), and RGO was spontaneously assembled on the metal foils. The metal oxides on RGO were carefully washed away with over $2 \mathrm{M} \mathrm{HCl}$ aqueous solution, followed with deionized water. The lyophilization was carried out to get the 3D RGO network. All the metal foils were purchased from Alfa Aesar with a purity of $>99.9 \%$. To accelerate the reaction and prevent the passivation effect due to the coating of the produced nonconductive metal oxides, a mild temperature of $60^{\circ} \mathrm{C}$ is also applied on demand.

Spontaneous deposition of RGO network on the surfaces of inert metals of Au, Ag, $\mathrm{Pt}$ and semiconducting Si (N-type). We first deposited a layer of $\mathrm{Cu}$ on one sides of $\mathrm{Au}, \mathrm{Ag}, \mathrm{Pt}$ foils, and $\mathrm{Si}$ wafer by vacuum sputtering, which were then exposure to $1 \mathrm{mg} / \mathrm{mL}$ GO solution for several hours. Apart from the Cu sides, RGO network was also spontaneously deposited on the $\mathrm{Cu}$-free sides of $\mathrm{Au}, \mathrm{Ag}, \mathrm{Pt}$ foils and $\mathrm{Si}$ wafer. The alternative method for depositing RGO network on the surface of inert metals and $\mathrm{Si}$ is covering them by $\mathrm{Cu}$ foil partly, followed by directly immersing them in $1 \mathrm{mg} / \mathrm{mL}$ GO solution for a certain time.

Spontaneous deposition of RGO network on the surfaces of $\mathrm{Au}, \mathrm{Ag}, \mathrm{Pt}$ nanostructures. The preparation of $\mathrm{Au}, \mathrm{Ag}$, Pt nanostructures with different size shape on $\mathrm{Cu}$ foils were carried out as described in our previous publications ${ }^{26,55}$. Briefly, on sides of the $\mathrm{Cu}$ foils were contacted with aqueous $0.01 \mathrm{M} \mathrm{HAuCl}_{4}, 0.02 \mathrm{M}$ $\mathrm{AgNO}_{3}$, and $0.01 \mathrm{M} \mathrm{H}_{2} \mathrm{PtCl}_{6}$ solutions for $1 \mathrm{~min}$, respectively, for the deposition of the nanostructured $\mathrm{Au}, \mathrm{Ag}$ or Pt with different sizes and shapes on $\mathrm{Cu}$ foils as shown in Supplementary Fig. S19-S22. Subsequently, $\mathrm{Cu}$ foils were washed with $\mathrm{HCl}$ solution and deionized water several times carefully before further experiments. For the formation of RGO network on the nanostructures, the $\mathrm{Cu}$ foils with nanostructured Au, Ag, Pt were exposed to $1 \mathrm{mg} / \mathrm{mL}$ GO solution for $8 \mathrm{~h}$, which were then washed by $\mathrm{HCl}$ solution, deionized water several times, characterized after lyophilization.

Spontaneous deposition of RGO network on indium-tin oxide (ITO) surface. ITO glass was half covered with $\mathrm{Cu}$ layer by vacuum sputtering, which was then exposed to $1 \mathrm{mg} / \mathrm{mL}$ GO solution for $12 \mathrm{~h}$ at room temperature (Supplementary Fig. S25). After that, the ITO was washed with deionized water slightly before lyophilization. Finally, the remained $\mathrm{Cu}$ was removed from the ITO glass carefully, and the region of RGO on ITO was still transparent due to the short reaction time.

Spontaneous deposition of RGO network on carbon film. We used the carbon film coated $\mathrm{Cu}$-based TEM grid as an example to demonstrate the effective deposition of RGO on carbon film. A carbon-coated Cu TEM grid was immersed in the $1 \mathrm{mg} / \mathrm{mL}$ GO solution for $10 \mathrm{~h}$, which was then washed with aqueous $\mathrm{HCl}$ and deionized water, followed by lyophilization before characterization.

Spontaneous deposition of RGO network on graphene film. A two step deposition of RGO was devised for this purpose (Supplementary Fig. S27\&28). The graphene film supported by $\mathrm{Cu}$ was first prepared by exposure of $\mathrm{Cu}$ foil to the $0.5 \mathrm{mg} / \mathrm{mL}$ $\mathrm{GO}$ solution for $10 \mathrm{~h}$, which was washed with $\mathrm{HCl}$ solution and deionized water several times carefully, followed by naturally drying to form a graphene film on $\mathrm{Cu}$. This $\mathrm{Cu}$ supported graphene film was then immersed in $1 \mathrm{mg} / \mathrm{mL}$ GO solution for $10 \mathrm{~h}$ for the further deposition of RGO on the preformed graphene film. The RGO on graphene film could be directly peeled off from the Cu foil or separated from the $\mathrm{Cu}$ foil by $1 \mathrm{M} \mathrm{FeCl}_{3}$ treatment for $0.5 \mathrm{~h}$. Finally, the 3D RGO assembled on graphene film was collected after washing with deionized water and lyophilization.

Preparation of graphene microtubes ( $\mu \mathrm{GTs}$ ). The formation of $\mu \mathrm{GT}$ s is schematically shown in Supplementary Fig. S29. A Cu wire was immersing into $1 \mathrm{mg} /$ $\mathrm{mL}$ GO solution for $10 \mathrm{~h}$ to allow the assembly of RGO along the $\mathrm{Cu}$ wire. Then the wet graphene enwrapped $\mathrm{Cu}$ wire was taken out from the solution, and densely packed graphene layer surrounding $\mathrm{Cu}$ wire was formed after drying naturally. Subsequently, $\mu \mathrm{GT}$ s with a diameter depending on the supporting $\mathrm{Cu}$ wire were collected after etching $\mathrm{Cu}$ wire in an aqueous $2.5 \mathrm{M} \mathrm{FeCl}_{3}$ solution containing $0.5 \mathrm{M}$ $\mathrm{HCl}$ and thoroughly washing with distilled water. The $\mathrm{Cu}$ wire can not only reduce the GO to RGO, but also act as the support during the drying process. The $\mu \mathrm{GT}$ s with diameters of $c a .100$ (Supplementary Fig. S30) and $40 \mu \mathrm{m}$ (Fig. 4b) were produced by choosing the $\mathrm{Cu}$ wires with corresponding diameters as supporting core.

The preparation of Y-shaped $\mu \mathrm{GT}$ with spiral structure, four-way pipe and multichannel network of graphenes. Based on the formation of $\mu$ GTs mentioned above, different configurations of $\mathrm{Cu}$ wires (Supplementary Fig. S32\&33), and $\mathrm{Cu}$ or Fe meshes (Supplementary Fig. S34) were exposed to the $1 \mathrm{mg} / \mathrm{mL}$ GO solution to allow the formation of $\mathrm{Y}$-shaped $\mu \mathrm{GT}$ with spiral structure, four-way pipe and multichannel network of graphenes.

The preparation of RGO pattern on Pt foil. As presented in Supplementary Fig. S35, by pressing a $\mathrm{Cu}$ grid $(200 \mu \mathrm{m}$ in side length) on Pt foil and then exposing them to the $1 \mathrm{mg} / \mathrm{mL}$ GO suspension for $12 \mathrm{~h}$, we can achieve the RGO patterns on $\mathrm{Pt}$ foil after removing the $\mathrm{Cu}$ grid. The region-confined 3D graphene network remains well with freeze-drying (Fig. $4 i \& j$ ). 
The preparation of region-defined pattern of $\mathrm{RGO}$ on $\mathrm{SiO}_{2}$. $\mathrm{Cu}$ islands on $\mathrm{SiO}_{2}$ were first obtained by vacuum sputtering with a $\mathrm{Cu}$ grid $(400 \mu \mathrm{m}$ in side length) as mask (Supplementary Fig. S36), which was then immersed in $1 \mathrm{mg} / \mathrm{mL} \mathrm{GO} \mathrm{for} 12 \mathrm{~h}$ to assembly the RGO on the Cu deposited regions. 3D porous RGO patterns on $\mathrm{SiO}_{2}$ ware produced by freeze-drying procedure. The patterns of RGO film were obtained with naturally drying.

Fabrication of 3D RGO/Cu electrode. Firstly, the trimmed $\mathrm{Cu}$ foil with circular shape was immersed in $1 \mathrm{mg} / \mathrm{mL} \mathrm{GO}$ solution for $10 \mathrm{~h}$ for the spontaneous assembly of RGO on $\mathrm{Cu}$ foil. After slightly washing with aqueous $\mathrm{HCl}$, the sample was freezedried to keep 3D pore-interconnected graphene structure on $\mathrm{Cu}$, which was directly used as working electrode in LIBs without need of any ancillary materials such as binders and conductive additives required for the conventional LIB fabrication.

LIB investigation. The as-obtained sample was directly used as working electrode without any ancillary materials for cell assembly. A lithium foil was used as the counter electrode and a solution of $1 \mathrm{M} \mathrm{LiPF}_{6}$ in ethylene carbonate (EC)/dimethyl carbonate (DMC)/diethyl carbonate (DEC) $(1: 1: 1$ in volume) was used as electrolyte. The assembly of the cells was performed in an argon-filled glove box. The cells were then aged for $8 \mathrm{~h}$ before measurement. The cycling tests were charged and discharged using an LAND CT2001A test station. The impendence spectra were obtained by applying a sine wave with amplitude of $5 \mathrm{mV}$ over the frequency range from $100 \mathrm{kHz}$ to $0.01 \mathrm{~Hz}$. The weights of graphenes on $\mathrm{Cu}$ foil were determined by measuring more than 10 parallel samples under the same condition. A mean value was used in this study.

Characterization. The morphology of the samples was determined by FET TECNAI F30 high resolution transmission electron microscopy (HR-TEM) at an acceleration voltage of $200 \mathrm{kV}$. Field-emission scanning electron microscope (FE-SEM) images and EDS data of the samples were taken on JSM-7001F SEM unit. X-ray diffraction (XRD) patterns were obtained by using a Netherlands 1,710 diffractometer with a $\mathrm{Cu}$ $\mathrm{K} \alpha$ irradiation source $(\lambda=1.54 \AA)$, and a self-calibration process was performed with a $\mathrm{SiO}_{2}$ internal standard sample prior to target measurement. X-ray Photograph electron spectroscopy (XPS) data were obtained with an ESCALab220i-XL electron spectrometer from VG Scientific using $300 \mathrm{~W}$ AlK $\alpha$ radiation. The base pressure was about $3 \times 10^{-9}$ mbar. The binding energies were referenced to the $\mathrm{C} 1 \mathrm{~s}$ line at $284.8 \mathrm{eV}$ from adventitious carbon.

1. Sun, Y. Q., Wu, Q. \& Shi, G. Q. Graphene based new energy materials. Energy Environ. Sci. 4, 1113-1132 (2011).

2. Huang, X., Qi, X. Y., Boey, F. \& Zhang, H. Graphene-based composites. Chem.Soc. Rev. 41, 666-686 (2012).

3. Chen, D., Tang, L. H. \& Li, J. H. Graphene-based materials in electrochemistry. Chem. Soc. Rev. 39, 3157-3180 ( 2010).

4. Shao, Y. Y. et al. Graphene based electrochemical sensors and biosensors: a review. Electroanal. 22, 1027-1036 (2010).

5. Chen, H., Muller, M. B., Gilmore, K. J., Wallace, G. G. \& Li, D. Mechanical strong, electrically conductive, and biocompatible graphene paper. Adv. Mater. 20, 3557-3561 (2008).

6. Schwierz, F. Graphene transistors. Nat. Nanotechnol. 5, 487-496 (2010).

7. Park, S. J. \& Ruoff, R. S. Chemical methods for the production of graphenes. Nat. Nanotechnol. 4, 217-224 (2009).

8. Stankovich, S. et al. Synthesis of graphene-based nanosheets via chemical reduction of exfoliated graphite oxide. Carbon 45, 1558-1565 (2007).

9. Su, C. Y. et al. Highly efficient restoration of graphitic structure in graphene oxide using alcohol vapors. ACS Nano 4, 5285-5292 (2010).

10. Fan, X. B. et al. Deoxygenation of exfoliated graphite oxide under alkaline conditions: a green route to graphene preparation. Adv. Mater. 20, 4490-4493 (2008).

11. Park, S. et al. Aqueous suspension and characterization of chemically modified graphene sheets. Chem. Mater. 20, 6592-6594 (2008).

12. Gao, W., Alemany, L. B., Ci, L. J. \& Ajayan, P. M. New insights into the structure and reduction of graphite oxide. Nat. Chem. 1, 403-408 (2009).

13. Moon, I. K., Lee, J., Ruoff, R. S. \& Lee, H. Reduced graphene oxide by chemical graphitization. Nat. Commun. 1, 1 (2010).

14. Feng, H. B., Cheng, R., Zhao, X., Duan, X. F. \& Li, J. H. A low-temperature method to produce highly reduced graphene oxide. Nat. Commun. 4, 1539 (2013).

15. Mao, S., Pu, H. H. \& Chen, J. H. Graphene oxide and its reduction: modeling and experimental progress. RSC Adv. 2, 2643-2662 (2012)

16. Hu, C. G. et al. Newly-designed complex ternary Pt/PdCu nanoboxes anchored on three-dimensional graphene framework for highly efficient ethanol oxidation. Adv. Mater. 24, 5493-5498 (2012).

17. Wu, Z. S. et al. Three-dimensional graphene-based macro- and mesoporous frameworks for high-performance electrochemical capacitive energy storage. J. Am. Chem. Soc. 134, 19532-19535 (2012).

18. Zhao, Y. et al. A versatile, ultralight, nitrogen-doped graphene framework. Angew. Chem. Int. Ed. 51, 11371-11375 (2012).

19. Xu, Y. X., Sheng, K. X., Li, C. \& Shi, G. Q. Self-assembled graphene hydrogel via a one-step hydrothermal process. ACS Nano 4, 4324-4330 (2010).

20. Fan, Z. J. et al. Facile synthesis of graphene nanosheets via Fe reduction of exfoliated graphite oxide. ACS Nano 5, 191-198 (2011).
21. Fan, Z. J. et al. An environmentally friendly and efficient route for the reduction of graphene oxide by aluminum powder. Carbon 48, 1670-1692 (2010).

22. Cao, X. B. et al. Ambient fabrication of large-area graphene films via a synchronous reduction and assembly strategy. Adv. Mater. (2013), DOI: 10.1002/ adma.201300586.

23. Yin, M. et al. Copper oxide nanocrystals. J. Am. Chem. Soc. 127, 9506-9511 (2005).

24. Borgohain, K., Murase, N. \& Mahamuni, S. Synthesis and properties of $\mathrm{Cu}_{2} \mathrm{O}$ quantum particles. J. Appl. Phys. 92, 1292-1297 (2002).

25. Li, Y. R., Sheng, K. X., Yuan, W. J. \& Shi, G. Q. A high-performance flexible fibreshaped electrochemical capacitor based on electrochemically reduced graphene oxide. Chem. Commun. 49, 291-293 (2013).

26. Qu, L. T. \& Dai, L. M. Substrate-enhanced electroless deposition of metal nanoparticles on carbon nanotubes. J. Am. Chem. Soc. 127, 10806-10807 (2005)

27. Hu, C. G. et al. Graphene microtubings: controlled fabrication and site-specific functionalization. Nano Lett. 12, 5879-5884 (2012).

28. Zhao, Y. et al. Large-scale spinning assembly of neat, morphology-defined, graphene-based hollow fibers. ACS Nano 7, 2406-2412 (2013).

29. Novoselov, K. S. et al. Electric field effect in atomically thin carbon films. Science 306, 666-669 (2004).

30. Geim, A. K. Graphene: status and prospects. Science 324, 1530-1534 (2009).

31. Persson, K. et al. Lithium diffusion in graphitic carbon. J. Phys. Chem. Lett. 1, 1176-1180 (2010).

32. Uthaisar, C. \& Barone, V. Edge effects on the characteristics of Li diffusion in graphene. Nano Lett. 10, 2838-2842 (2010).

33. Hu, Y. S. et al. Synthesis of hierarchically porous carbon monoliths with highly ordered microstructure and their application in rechargeable lithium batteries with high-rate capability. Adv. Funct. Mater. 17, 1873-1878 (2007) .

34. Qie, L. et al. Nitrogen-doped porous carbon nanofiber webs as anodes for lithium ion batteries with a superhigh capacity and rate capability. Adv. Mater. 24, 2047-2050 (2012).

35. Wu, X., Liu, Q., Guo, Y. \& Song, W. Superior storage performance of carbon nanosprings as anode materials for lithium-ion batteries. Electrochem. Commun 11, 1468-1471 (2009).

36. Li, G. D., Xu, L. Q., Hao, Q., Wang, M. \& Qian, Y. T. Synthesis, characterization and application of carbon nanocages as anode materials for high-performance lithium-ion batteries. RSC Adv. 2, 284-291 (2012).

37. Habazaki, H., Kiriu, M. \& Konno, H. High rate capability of carbon nanofilaments with platelet structure as anode materials for lithium ion batteries. Electrochem. Commun. 8, 1275-1279 (2006).

38. Shen, J. M. \& Feng, Y. T. Formation of flower-like carbon nanosheet aggregations and their electrochemical application. J. Phys. Chem. C 112, 13114-13120 (2008).

39. Zhang, J.et al.CNFs@CNTs: superior carbon for electrochemical energy storage. Adv. Mater. 20, 1450-1455 (2008).

40. Wu, Z. S., Ren, W. C., Xu, L., Li, F. \& Cheng, H. M. Doped graphene sheets as anode materials with superhigh rate and large capacity for lithium ion batteries. ACS Nano 7, 5463-5471 (2011).

41. Wang, Z. L., Xu, D., Wang, H. G., Wu, Z. \& Zhang, X. B. In situ fabrication of porous graphene electrodes for high-performance energy storage. ACS Nano 7 , 2422-2430 (2013).

42. $\mathrm{Hu}$, L. et al. $\mathrm{CoMn}_{2} \mathrm{O}_{4}$ spinel hierarchical microspheres assembled with porous nanosheets as stable anodes for lithium-ion batteries. Sci. Rep. 2, 986-993 (2012)

43. Reddy, A. L. M., Shaijumon, M. M., Gowda, S. R. \& Ajayan, P. M. Coaxial $\mathrm{MnO}_{2} /$ carbon nanotube array electrodes for high-performance lithium batteries. Nano Lett. 9, 1002-1006 (2009).

44. Wei, W. et al. 3D graphene foams cross-linked with pre-encapsulated $\mathrm{Fe}_{3} \mathrm{O}_{4}$ nanospheres for enhanced lithium storage. Adv. Mater. (2013), DOI: 10.1002/ adma.201300445

45. Hu, C. G. et al. Highly nitrogen-doped carbon capsules: scalable preparation and high-prformance applications in fuel cells and lithium ion batteries. Nanoscale 5, 2726-2733 (2013)

46. Xue, Y. H. et al. Oxidizing metal ions with graphene oxide: the in situ formation of magnetic nanoparticles on self-reduced graphene sheets for multifunctional applications. Chem. Commun. 47, 11689-11691 (2011).

47. Pei, S. F., Zhao, J. P., Du, J. H., Ren, W. C. \& Cheng, H. M. Direct reduction of graphene oxide films into highly conductive and flexible graphene films by hydrohalic acids. Carbon 48, 4466-4474 (2010).

48. Liu, L. et al. Amorphous structural models for graphene oxides. Carbon $\mathbf{5 0}$ 1690-1698 (2012).

49. Dreyer, D. R., Park, S. J., Bielawski, C. W. \& Ruoff, R. S. The chemistry of graphene oxide. Chem. Soc. Rev. 39, 228-240 (2010).

50. Perdew, J. P., Burke, K. \& Ernzerhof, M. Generalized gradient approximation made simple. Phys. Rev. Lett. 77, 3865-3868 (1996).

51. Kresse, G. \& Furthmüller, J. Efficient iterative schemes for ab initio total-energy calculations using a plane-wave basis set. Phys. Rev. B 54, 11169-11186 (1996).

52. Kresse, G. \& Furthmüller, J. Efficiency of ab-initio total energy calculations for metals and semiconductors using a plane-wave basis set. Comput. Mat. Sci. 6-15 (1996).

53. Li, Y. et al. An electrochemical avenue to green-luminescent graphene quantum dots as potential plectron-acceptors for photovoltaics. Adv. Mater. 23, 776-780 (2011). 
54. Li, Y. et al. Nitrogen-doped graphene quantum dots with oxygen-rich functional groups. J. Am. Chem. Soc. 134, 15-18 (2012).

55. Qu, L. T., Dai, L. M. \& Osawa, E. Shape/size-controlled syntheses of metal nanoparticles for site-selective modification of carbon nanotubes. J. Am. Chem. Soc. 128, 5523-5532 (2006).

\section{Acknowledgements}

This work is sponsored by National Basic Research Program of China (2011CB013000), NSFC (No. 21004006, 21174019, 51161120361), Fok Ying Tong Education Foundation (No. 131043), the 111 Project B07012, and research foundation for the doctoral program of higher education of China (20101101120036).

\section{Author contributions}

L.Q. conceived and designed the experiments. C.H., X.Z. and Y.Z. prepared the samples and performed characterization. L.L performed the computer calculation. L.Q. discussed with
L.J. for the experimental design and results. L.Q., C.H. and L.L. were mainly responsible for preparing the manuscript with further inputs from other authors. All the authors discussed the results and commented on the manuscript.

\section{Additional information}

Supplementary information accompanies this paper at http://www.nature.com/ scientificreports

Competing financial interests: The authors declare no competing financial interests.

How to cite this article: Hu, C.G. et al. Spontaneous Reduction and Assembly of Graphene oxide into Three-Dimensional Graphene Network on Arbitrary Conductive Substrates. Sci. Rep. 3, 2065; DOI:10.1038/srep02065 (2013).

(c) (i) $(-)$ This work is licensed under a Creative Commons Attribution-

NC ND NonCommercial-NoDerivs 3.0 Unported license. To view a copy of this license, visit http://creativecommons.org/licenses/by-nc-nd/3.0 